\section{NIV in clinical practice: view from the frontline}

\section{P1 NON-INVASIVE VENTILATION IN MOTOR NEURONE DISEASE: THE UTILITY OF THE EPWORTH SLEEPINESS SCALE AS AN OUTCOME MEASURE}

${ }^{1} \mathrm{LH}$ Piggin, 'EW Thornton, ${ }^{2} \mathrm{RM}$ Angus, ${ }^{2} \mathrm{~B}$ Chakrabarti, ${ }^{3} \mathrm{CA}$ Young. 'School of Psychology, University of Liverpool, Liverpool, UK; ${ }^{2}$ Aintree Chest Centre, University Hospital Aintree, Liverpool, UK; ${ }^{3}$ Walton Centre for Neurology and Neurosurgery, Liverpool, UK

\section{doi:10.1136/thx.2009.127126a}

Introduction: The Epworth Sleepiness Scale (ESS) is used to quantify changes in daytime somnolence. It is not known whether quantitative changes in ESS scores accurately reflect qualitative experiences of patients with motor neurone disease (MND) following initiation of non-invasive ventilation (NIV) in terms of perceived changes in sleep quality, fatigue and daytime somnolence. Methods: This study, comprising both quantitative and qualitative methods, assessed 7 MND patients ( 6 men; mean age 66 years) immediately prior to NIV commencement and again 3 months post NIV initiation. Each assessment included overnight pulse oximetry, the ESS and one semi-structured interview. Interviews underwent thematic analysis before comparison with quantitative data.

Results: Pre-NIV, 3 patients scored $\geqslant 10$ on ESS (range 2-17; $M=8.0$ ). Pre-NIV ESS scores correlated significantly with percentage time $<90 \%$ saturation in pre-NIV oximetry $(M=21.22 \%$, $\mathrm{SD}=22.06 ; \mathrm{r}=0.786, \mathrm{p}=0.036)$. However, ESS scores did not appear to correspond to individual qualitative experiences, underestimating symptom severity. The majority experienced disturbed sleep, fatigue and daytime somnolence that often caused low mood and negative illness perception. Post-NIV, ESS scores (range 1-12; $M=6.29$ ) did not differ significantly from pre-NIV scores ( $t$ $(6)=0.61, p=0.56)$ and did not correlate with any post-NIV oximetry markers. The trend in ESS scores towards decreased sleepiness $(M=0.29, \mathrm{SD}=7.65)$ following NIV initiation did significantly correlate with the change in percentage time spent $<90 \%$ saturation $(\mathrm{M}=16.8, \mathrm{SD}=13.24 ; \mathrm{r}=0.841, \mathrm{p}=0.018)$. Qualitatively, patients reported substantial improvement in sleep quality and reductions in daytime somnolence beyond those suggested by change in ESS scores. For the majority, qualitative changes following NIV initiation were significant enough to improve mood, illness perception and expectations of life with MND.

Conclusion: ESS scores correlated with some physiological markers of nocturnal hypoventilation prior to NIV but underestimated the impact of daytime somnolence, failing to capture the magnitude of positive changes perceived as a result of NIV use. This suggests that the ESS requires substantiation by qualitative data if it is to be used as an effective outcome measure in research addressing NIV use in this population.

\section{P2 PREDICTORS OF SUCCESSFUL DOMICILIARY NON-INVASIVE VENTILATION FOR MOTOR NEURONE DISEASE IN A DISTRICT GENERAL HOSPITAL}

RJ Parker, GV Robinson, AD McGown, CWH Davies. Royal Berkshire NHS Foundation Trust, Reading, UK

\section{doi:10.1136/thx.2009.127126b}

Introduction and Objectives: Respiratory muscle weakness is common in motor neurone disease (MND). Non-invasive ventilation (NIV) controls nocturnal hypoventilation improving daytime sleepiness, quality of life and may provide survival benefits, particularly in non-bulbar MND. The American Academy of Neurologists guidelines provide standards of care for MND including vigilance for symptoms of nocturnal hypoventilation and serial pulmonary function testing. Vital capacity (VC) $<50 \%$ predicted or $<1.51$ should prompt assessment for NIV. Assessment methods and predictors of success (defined as NIV use for $>3$ months) were analysed to enable recommendations for service improvement to be made.

Methods: Review of clinical notes for $21 / 23$ patients with MND beginning NIV in 2001-9.

Results: Nine patients were male, mean age commencing NIV was $65.3 \pm 7.7$ years and 11 had bulbar MND. A median of one respiratory clinic was attended before NIV was commenced. Eight needed urgent NIV. Mean NIV use after successful set-up was $18.4 \pm 12.4$ months vs $1.3 \pm 1.3$ months in the "failure" group (table 1). Symptoms of hypoventilation and daytime sleepiness were incompletely documented, particularly in neurology clinic letters, and Epworth score was never measured. Oxygen saturation, $\mathrm{VC}$ and arterial blood gases were measured in all respiratory clinic attendees. $14 / 21$ patients had a daytime $\mathrm{PCO}_{2}>6.0 \mathrm{kPa}$. Ten underwent sleep studies.

Conclusions: Many patients were referred late in their disease trajectory. Most were seen only once in the respiratory clinic before needing to start NIV. On first contact with respiratory medicine, $57 \%$ had a VC below which NIV should be considered, with the majority in daytime ventilatory failure. Those successfully starting NIV had better VC, non-bulbar MND, elective set-up and less chronic ventilatory failure evidenced by base excess and bicarbonate values than those discontinuing NIV. These factors were constant over the time period examined. Home ventilatory support for neuromuscular disease is a developing area of respiratory medicine, increasingly performed outside tertiary ventilation units Neurological colleagues need to recognise the indications for NIV, ensure timely referral and elective initiation of ventilatory support to improve the success rates.

Abstract P2 Table 1 Factors affecting success of NIV

\begin{tabular}{lcl}
\hline & Success & Failure \\
\hline Number of patients & 8 & 13 \\
Age starting NIV (years) & $65.5 \pm 6.3$ & $65.7 \pm 8.3$ \\
$\mathrm{BMI}\left(\mathrm{kg} / \mathrm{m}^{2}\right.$ ) & $26.1 \pm 7.4$ & $22.6 \pm 5.3$ \\
Bulbar MND & 1 & $10^{*}$ \\
Urgent NIV & 2 & 6 \\
VC (I) & $2.1 \pm 0.7$ & $1.3 \pm 0.7^{*}$ \\
PEG in situ & 2 & 7 \\
VC $<1.5$ I or $<50 \%$ predicted & 2 & $10^{*}$ \\
Pco 2 (kPa) & $7.7 \pm 2.8$ & $7.3 \pm 2.3$ \\
Base excess (mmol/l) & $3.4 \pm 4.1$ & $6.3 \pm 4.3$ \\
Bicarbonate (mmol/l) & $27.0 \pm 2.8$ & $30.3 \pm 1.3^{*}$ \\
Most common reasons for stopping NIV & Death 5 & Not tolerated 6, death 4 \\
\hline
\end{tabular}

$\mathrm{BMI}$, body mass index; MND, motor neurone disease; NIV, non-invasive ventilation; $\mathrm{PCO}_{2}$ carbon dioxide tension; VC, vital capacity. ${ }^{*} \mathrm{p}<0.05$.

\section{P3 OUTCOMES OF COPD PATIENTS REQUIRING RECURRENT NIV FOR HYPERCAPNIC RESPIRATORY FAILURE}

A Ponnuswamy, A Abdul, RM Angus. Aintree Chest Centre, University Hospital Aintree, Liverpool, UK

doi:10.1136/thx.2009.127126c

Non-invasive ventilation (NIV) improves the outcome in exacerbations of chronic obstructive pulmonary disease (COPD) with 
decompensated type 2 respiratory failure. However, the data on the benefit of NIV in patients requiring repeated acute NIV is less clear. We followed the clinical course in a cohort collected over 3 years from the index hospital admission.

Patient data were retrieved from patients admitted to the respiratory support unit from May 2005 to November 2008 who are logged on admission. All patients with COPD and two or more admissions requiring acute NIV were included. Statistical significance was determined by logistic regression analysis and $\chi^{2}$ tests.

50 patients met the criteria. $31(63 \%)$ were women. The mean age was 67 years (range 36-88); 11 (22\%) are still alive. Mean forced expiratory volume in $1 \mathrm{~s}\left(\mathrm{FEV}_{1}\right)$ was 0.691 (range $\left.0.36-1.5\right) .47$ (94\%) patients had an estimated WHO performance status of $>3.35(71 \%)$ patients were on home oxygen, 38 (76\%) were on LTOT and 7 (14\%) were established on home NIV after the second episode of acute NIV. 35 (70\%) patients were ex-smokers and $15(30 \%)$ were current smokers. Overall, 22 (44\%) patients had a third NIV, 11 (22\%) had a fourth NIV, 5 (10\%) had a fifth NIV and 1 patient went on to have seven episodes of NIV. Of those alive, 11 had a second episode of NIV and 5 had more than three NIV episodes. There was a significant difference in 30-day survival between those receiving a second NIV episode (50\%;11 vs 22) and those receiving a third NIV episode $(29 \% ; 5$ vs 17$)(p<0.003)$.

As expected, patients with COPD requiring repeated support with acute NIV have severe disease, marked functional limitation and poor outlook. This prospective evaluation suggests that there is a diminishing return after the third NIV episode. This should be considered with patients when undertaking advanced planning regarding future exacerbations.

\section{P4 NIV IN COPD PATIENTS WITH ACUTE HYPERCAPNIC RESPIRATORY FAILURE: OUTCOME IN PATIENTS WITH RADIOLOGICAL EVIDENCE OF CONSOLIDATION}

SR Henderson, TJ Tull, CR Sander, JL Brown. Departments of Acute and Respiratory Medicine, Hammersmith Hospital, Imperial College Healthcare NHS Trust, London, UK

\section{doi:10.1136/thx.2009.127126d}

Introduction: BiLevel Positive Airway Pressure Ventilation (BiPAP) has a beneficial role in the management of acute type II respiratory failure secondary to chronic obstructive pulmonary disease (COPD). Studies have shown that pneumonia predicts failure of non-invasive ventilation (NIV) therapy and ready access to invasive ventilation is important.

Objective: To evaluate the outcome of BiPAP ventilation in COPD patients with acute type II respiratory failure and radiological evidence of consolidation.

Methods: Patients with COPD admitted to the AMU with acute type II respiratory failure treated with BiPAP ventilation were retrospectively evaluated between May 2005 and November 2008. Their clinical characteristics, chest radiographic appearances, length of hospitalisation, mortality and intubation rates were recorded. Arterial blood gas analysis pre-NIV, $1-2 \mathrm{~h}$ and $4-6 \mathrm{~h}$ after BiPAP ventilation allowed subsequent calculation of the $\mathrm{A}-\mathrm{a}$ gradient for each time point.

Results: 102 COPD patients (63 women, 39 men) required BiPAP ventilation for acute type II respiratory failure between May 2005 and November 2008. Mean \pm SD patient age was $71.7 \pm 11.1$ years. Radiological evidence of consolidation on the chest radiograph was present in $35 \%(n=36)$ of patients. Significant reverse correlation with both pre-NIV and post-NIV A-a gradients was demonstrated in patients with pneumonia $(\mathrm{r}=-0.2, p=0.03 / \mathrm{r}=-0.25, \mathrm{p}=<0.01$, respectively). In addition, correlation between length of hospitalisation and $\mathrm{A}-\mathrm{a}$ gradient pre-NIV and post-NIV was observed $(r=0.31$, $\mathrm{p}=<0.01, \mathrm{r}=0.2, \mathrm{p}=<0.05$, respectively). Average length of hospitalisation was 20 (1-191) days. Importantly, the presence of consolidation did not show correlation with NIV treatment failure, intubation or death. Intubation and escalation to intensive care was undertaken in 6 patients. Mortality in patients with consolidation was $10 \%$ compared with $11 \%$ in patients without radiological consolidation.

Conclusions: In our retrospective analysis, radiological consolidation was not associated with NIV treatment failure or increased mortality, but did correlate with increased length of stay. Pneumonia is a risk factor for NIV treatment failure and early intubation and invasive ventilation remain essential for patients who fail to tolerate NIV. However, in COPD patients in whom NIV is the ceiling of therapy, the presence of radiological consolidation should not prevent the trial of NIV.

\section{P5 IMPACT OF DOMICILIARY NON-INVASIVE VENTILATION (NIV) SERVICE IN CHRONIC VENTILATORY DISORDER ON HOSPITALISATION AND QUALITY OF LIFE: A GRIMSBY EXPERIENCE}

A Dwarakanath, H O'Flynn. Diana, Princess of Wales Hospital, Grimsby, UK

\section{doi:10.1136/thx.2009.127126e}

Introduction: Respiratory muscle weakness is inevitable in many congenital neuromuscular chest wall disorders and is the most common cause of recurrent chest infections requiring hospital admissions. The benefits of non-invasive ventilation (NIV) in these patients are intuitive, but have not been fully delineated in randomised trials because ethical considerations have precluded the use of a control group. The long-term outcome of NIV in these conditions has shown improved gas exchange and enhanced healthrelated quality of life.

Aim: To determine whether adequate domiciliary NIV in chronic ventilatory disorders improves the performance status and has an impact on hospitalisation and length of stay.

Method: Eleven patients on domiciliary NIV for more than 12 months with chronic ventilatory disorders were included (7 men). The WHO performance status was assessed pre- and postNIV. The number of hospital admission rates including the number of days spent in the hospital was obtained.

Results: Pre- and post-NIV WHO performance status were (1-2, $2-2,3-6,4-1)$ and $(1-2,2-7,3-2,4-0)$, respectively. The age distribution was $<30$ years (1), 30-60 years (2), >60 years (8). The causes of ventilatory failure were kyphoscoliosis (3), motor neurone disease (2), right hemidiaphragm weakness (1), thoracoplasty (2), bilateral diaphragmatic paralysis with myasthenia gravis (1), obesity hypoventilation (1), severe COPD (1). The types of ventilators used were VIVO 40 (1), NIPPV 2 (2), SYNCHRONY (7) and S/T 30 (1). The number of pre- and postNIV hospital admission rates was 29 and 7 times, respectively. The number of hospital days spent pre- and post-NIV were 292 days (ITU, 46; HDU, 66; ward, 180) and 57 days (ITU, 0; HDU, 16; ward, 41), respectively. The post-NIV hospital days were for retitration and other medical co-morbidities. Patients were monitored on a regular basis with overnight oximetry, early morning arterial blood gases and capnography while on domiciliary NIV and were also reviewed in the NIV clinics. Nine patients showed evidence of adequate nocturnal ventilation and experienced better quality of life.

Conclusion: Domiciliary NIV in chronic ventilatory disorders improves quality of life, performance status and nocturnal ventilation with fewer hospital admissions which is cost effective. 


\section{P6 CHANGES IN PULMONARY FUNCTION TESTS FOLLOWING INITIATION OF NON-INVASIVE VENTILATION IN PATIENTS WITH TYPE 2 RESPIRATORY FAILURE SECONDARY TO OBESITY}

'S Gledhill, ${ }^{2}$ TW Felton, ${ }^{1} \mathrm{~S}$ Johnson, ${ }^{1} \mathrm{~N}$ Clayton, ${ }^{2} \mathrm{AM}$ Bentley. ${ }^{1}$ University Hospital of South Manchester, Manchester, UK; ${ }^{2}$ The University of Manchester, Manchester, UK

\section{doi:10.1136/thx.2009.127126f}

Introduction and Objectives: Obesity is an increasingly common worldwide problem which frequently causes type II respiratory failure. Obesity hypoventilation syndrome (OHS) is characterised by reduced chest wall compliance, increased airway resistance and reduced tidal volume. An "overlap syndrome" occurs when the physiological changes of obesity combine with lung pathology, commonly chronic obstructive pulmonary disease (COPD). This pilot study aims to determine whether pulmonary function tests can identify patients with OHS from those with overlap syndrome and to investigate the physiological changes associated with commencing non-invasive ventilation (NIV).

Methods: Seven patients with OHS and 10 patients with overlap syndrome were recruited. Patients with overlap syndrome were identified by type II respiratory failure, body mass index (BMI) $>30 \mathrm{~kg} / \mathrm{m}^{2}$ and a clinical diagnosis in keeping with COPD. Prior to commencing NIV, ear lobe capillary blood gas samples and pulmonary function testing were prospectively recorded. Measurements were repeated during each patient's first follow-up appointments. The study was approved by the local research ethics committee.

Results: Four men and three women had OHS with a mean age of 56 years and five men and five women had an overlap syndrome with a mean age of 61 years. Both groups were markedly obese with higher BMI values recorded in the OHS group (table 1). Significant improvements in oxygen and carbon dioxide levels were seen following initiation of NIV in both groups. Prior to commencing NIV, the patients with OHS had higher recorded forced expiratory volume in $1 \mathrm{~s}\left(\mathrm{FEV}_{1}\right)$, forced vital capacity (FVC), $\mathrm{FEV}_{1} / \mathrm{FVC} \%$ and carbon monoxide lung transfer factor (TLCO) and lower recorded functional residual capacity (FRC) compared with patients with the overlap syndrome. Residual volume and FRC increased following treatment with NIV in both groups. Compliance was similar in both groups with a mean usage of $5.5 \mathrm{~h}$.

Conclusions: Nocturnal NIV for obesity-related type II respiratory failure improves gas exchange. Overlap syndrome can be identified by obstructive spirometry compared with restrictive spirometry

Abstract P6 Table 1 Patient data comparing physiological changes following initiation of NIV

\begin{tabular}{|c|c|c|c|c|}
\hline \multirow[b]{2}{*}{ Characteristic } & \multicolumn{2}{|c|}{ Pure obesity } & \multicolumn{2}{|c|}{ Overlap syndrome } \\
\hline & Pre-NIV & Post-NIV & Pre-NIV & Post-NIV \\
\hline BMI $\left(\mathrm{kg} / \mathrm{m}^{2}\right)$ & 54 & 50 & 43 & 39 \\
\hline Weight (kg) & 146 & 134 & 112 & 103 \\
\hline $\mathrm{P}_{2}(\mathrm{kPa})$ & 6.2 & 8.34 & 6.6 & 7.2 \\
\hline $\mathrm{PcO}_{2}(\mathrm{kPa})$ & 8.4 & 6.0 & 7.8 & 6.0 \\
\hline $\mathrm{FEV}_{1}(\%$ pred $)$ & 73.0 & 75.0 & 40.6 & 53.1 \\
\hline FVC (\% pred) & 79.0 & 85.0 & 56.0 & 72.9 \\
\hline $\mathrm{FEV}_{1} / \mathrm{FVC}(\%)$ & 75.9 & 71.4 & 58.7 & 59.1 \\
\hline FRC (\% pred) & 66.0 & 77.8 & 97.9 & 112.3 \\
\hline TLC (\% pred) & 81.0 & 85.2 & 81.1 & 94.4 \\
\hline RV (\% pred) & 86.6 & 92.0 & 118.4 & 130.7 \\
\hline TLco (\% pred) & 97.2 & 91.5 & 74.3 & 69.2 \\
\hline Kco (\% pred) & 123.5 & 113.2 & 107.5 & 99.5 \\
\hline
\end{tabular}

$\mathrm{BMI}$, body mass index; $\mathrm{FEV}_{1}$, forced expiratory volume in $1 \mathrm{~s}$; FRC, functional residual capacity; FVC, forced vital capacity; Kco, carbon monoxide transfer coefficient; NIV, noninvasive ventilation; $\mathrm{PCO}_{2}, \mathrm{PO}_{2}$, carbon monoxide and oxygen tensions; $\mathrm{RV}$, residual volume; TLC, total lung capacity; TLCO, carbon monoxide lung transfer factor. seen in OHS. Increased static lung volumes following NIV suggest that recruitment of lung units may, at least in part, explain the resolution of type II respiratory failure.

\section{P7 NON-INVASIVE VENTILATION TRAINING : A SIMULATED APPROACH FOR HEALTHCARE PROFESSIONALS}

SG McQueen, M Dickinson, M Pimblett. Lancashire Teaching Hospitals NHS Trust, Preston, UK

\section{doi:10.1136/thx.2009.127126g}

Introduction: Simulation-based training is a technique which facilitates a structured learning experience that replicates real-life situations and occurrences in an endeavour to accelerate the development of expertise in a given field, and has increased significantly in recent years. This has coincided with the increased use of non-invasive ventilation (NIV) as a treatment option for various conditions. Training staff to become confident in using NIV currently takes up to 4 months, depending on the number of patients requiring NIV to which the staff become exposed. The National COPD Audit 2008 highlighted the difficulties in training of staff.

Method: A training day for healthcare professionals (HPC) with a special interest in NIV was formulated. The course covered various aspects of NIV and, for the first time, the use of human patient simulation (HPS) was integrated into the teaching programme. The use of intermediate level HPS not only allowed participants to examine and respond to a "real person", but also allowed them to assemble and apply NIV in order to manage patients in real time in a simulated clinical environment which was not too dissimilar to their normal working area.

Results: Six staff were recruited to the trial programme with varying degrees of knowledge and experience in using NIV and arterial blood gas interpretation. Three staff had very limited knowledge of NIV application and were not confident to initiate or modify any treatment plans. Upon completion of the training day, all staff had been given the opportunity to implement and adjust NIV management on the simulated patient. The three staff members who previously had little experience of NIV were able to initiate and modify NIV in an appropriate manner on actual patients within 2 weeks of completing the day.

Conclusion: Although this is only a small cohort of staff, the evident reduction in training time and thus the potential to increase the manpower to care for NIV-dependent patients with efficiency and safety suggests that this method of training should be expanded and developed further

\section{P8 AN AUDIT OF DECISION MAKING WHEN COMMENCING AND DURING BI-LEVEL POSITIVE AIRWAY PRESSURE VENTILATION IN A DISTRICT GENERAL SETTING}

R Francis, M Harley, A Wickham, JA Hughes. Kent and Sussex Hospital, Tunbridge Wells, UK

doi:10.1136/thx.2009.127126h

Introduction and Objectives: Non-invasive ventilation (NIV) is a valuable treatment modality. The BTS published guidelines for the use of Bi-Level Positive Airway Pressure ventilation (BiPAP) for type II respiratory failure in October 2008. Each episode of treatment with BiPAP typically involves multiple adjustments based on patient response. This study assessed whether patients receive appropriate monitoring and adjustment of BiPAP according to clinical responses. The objective was to ascertain the accuracy of decision making by different grades of medical and nursing staff against a standard of the BTS guidelines. 


\section{Decisions made about BiPAP prescriptions by practitioner grade}

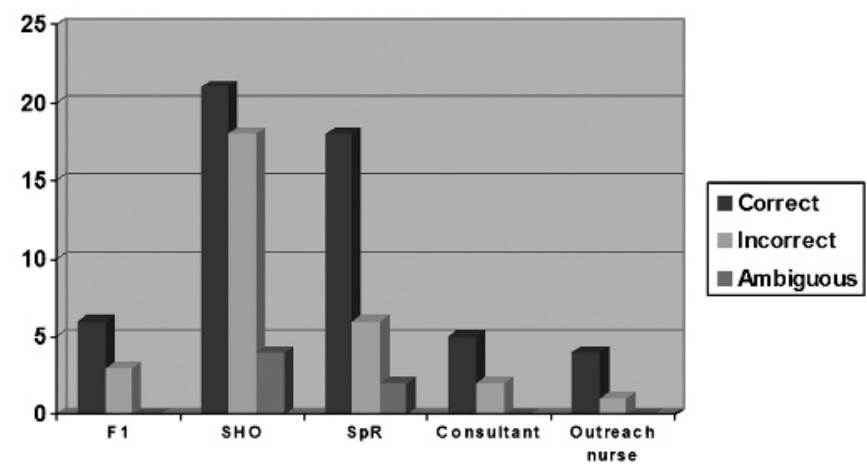

Abstract P8 Figure 1 Decisions made about BiPAP prescriptions by practitioner grade.

\begin{abstract}
Methods: Patients were in non-critical care environments in a district general hospital with an established NIV service. Arterial blood gases (ABGs) were recorded and each decision based on them was compared against the action suggested by BTS guidelines. The decision to start BiPAP and all adjustments were categorised by trained practitioners into one of three outcomes: "clearly correct", "clearly incorrect" and "ambiguous".

Results: 90 individual decisions in 23 patients were audited over a 2month period. Documentation was poor and key information suggested by the BTS guidelines was not recorded. A 4-6 h ABG was taken from only $65 \%$ of patients. Mean time to third ABG was 13.6 h. $60 \%$ of the 90 decisions audited were deemed correct, $33 \%$ incorrect and $7 \%$ were ambiguous. $10 \%$ of decisions were made by FY1 doctors. Registrars, consultants and outreach nurses were involved in decision making. Senior house officers (SHOs) made up of FY2-CT2 doctors made $47 \%$ of decisions, more than any other group. However, $42 \%$ of their decisions were incorrect. All other groups performed significantly better (fig 1).

Conclusions: The decision-making process during BiPAP therapy is not often audited. SHOs make more adjustments to BiPAP than other groups in the hospital but also make more errors. FY1s seek advice and SpRs have more experience. We have since organised training sessions for junior members of staff and are introducing a prescription sheet with detailed advice in line with BTS guidelines. We will be re-auditing a new cohort of staff at the same stage in their training as the group examined in this audit.
\end{abstract}

\section{P9 DOMICILIARY VENTILATOR INITIATION: SUITABLE FOR DAY CASE?}

E Sayell, BV Prathibha. William Harvey Hospital, Ashford, UK

\section{doi:10.1136/thx.2009.127126i}

Background: Patients with chronic type 2 respiratory failure due to kyphoscoliosis, obesity hypoventilation or neuromuscular disorders benefit from domiciliary nocturnal ventilatory support. This usually entails admission of the patient to the ward and a stay of at least 3 days to ensure patient education and comfort. We have piloted a day case service whereby selected patients are admitted as day cases to our ambulatory ward and initiated on domiciliary ventilatory support.

Methods: Patients with chronic type 2 respiratory failure are assessed for suitability of domiciliary ventilation in the clinic.
Abstract P9 Table 1 Characteristics of patients using domiciliary ventilation

\begin{tabular}{lllll}
\hline & Kyphoscoliosis & $\begin{array}{l}\text { Neuromuscular } \\
\text { disorder }\end{array}$ & $\begin{array}{l}\text { Obesity } \\
\text { hypoventilation }\end{array}$ & COPD \\
\hline Men & 1 & 5 & 9 & 5 \\
Women & 4 & 3 & 10 & 5 \\
\hline
\end{tabular}

COPD, chronic obstructive pulmonary disease.

This includes confirmation of diagnosis, overnight sleep study, blood gases and lung function. Those deemed suitable for domiciliary ventilatory support are then brought in as day cases to a nurse-led clinic at the ambulatory care unit at our hospital. They are asked to present themselves at $09.00 \mathrm{~h}$ and to undergo repeat blood gas measurements. They are then educated about domiciliary ventilation and set up on the machines (Respironics $\mathrm{BiPAP}$ ). After an hour on the BiPAP, the blood gases are repeated. They are thus monitored for 5-6 h (maximum $8 \mathrm{~h}$ ) and then discharged with an instruction booklet on non-invasive ventilation. They are reviewed by the nurse specialist within a week and again within a month, then regularly every 6 months.

Results: 42 patients have been set up on domiciliary ventilation over the last 12 months. Their characteristics are shown in table 1.

Conclusion: Initiation of domiciliary ventilation can be done safely on an outpatient basis. It requires dedicated staff and facilities. This is preferred by patients and is both cost and clinically effective.

\section{P10 SEVERITY OF ACIDOSIS AT PRESENTATION IS NOT LINKED TO SURVIVAL TO HOSPITAL DISCHARGE IN PATIENTS WITH ACUTE TYPE 2 RESPIRATORY FAILURE}

H Rupani, H Artis, R Agrawala, V Jones, S Fletcher, KMA O'Reilly. Southampton University Hospitals Trust, Southampton, UK

\section{doi:10.1136/thx.2009.127126j}

Background: Non-invasive ventilation (NIV) has been shown to reduce mortality and intubation rates in patients with chronic obstructive pulmonary disease (COPD) and acute type 2 respiratory failure. However, the evidence supporting the use of NIV in patients with COPD and severe acidosis $(\mathrm{pH}<7.26)$ or those with an alternative diagnosis is less robust. Our anecdotal experience suggested that patients in these subgroups frequently benefit from NIV and we sought to study this.

Methods: A retrospective case note analysis was carried out for 100 patients admitted with decompensated type 2 respiratory failure. The medical high dependency unit has a high ratio of nursing staff to patients (1:2) and NIV management is guided by a standardised protocol.

Results: $55 \%$ of patients had an initial $\mathrm{pH}<7.26$ and the majority $(65 \%)$ had a performance status of 2 or more. Only $14 \%$ of patients were felt to be suitable for intubation; four patients required intubation. $72 \%$ of patients who received NIV with appropriate medical therapy survived until hospital discharge. COPD was the underlying diagnosis in $72 \%$ of patients; of these, $57 \%$ had an initial $\mathrm{pH}<7.26$ (table 1). The in-hospital mortality for patients with severe acidosis was similar to that for patients with $\mathrm{pH}>7.26(33 \%$ vs 30\%). This was also the case for patients with COPD where the mortality was $22 \%$ and $21 \%$ for patients with $\mathrm{pH}<7.26$ and $\mathrm{pH}>$ 7.26 , respectively. Mortality was significantly worse in patients who had an underlying diagnosis of pneumonia than in those with COPD (77\% vs $21 \%$, table).

Discussion and Conclusion: These data suggest that providing NIV in a closely monitored environment can be successful even in patients with COPD presenting with severe acidosis $(\mathrm{pH}<7.26)$. Frequently 


\section{Abstract P10 Table 1}

\begin{tabular}{lll}
\hline Diagnosis & No of patients & $\begin{array}{c}\text { In-hospital } \\
\text { mortality }\end{array}$ \\
\hline COPD (all) & 72 & $15(21 \%)$ \\
${ }^{*} \mathrm{pH}>7.26$ & $29(40 \%)$ & $6(21 \%)$ \\
${ }^{*} \mathrm{pH}<7.26$ & $41(57 \%)$ & $9(22 \%)$ \\
Pneumonia & 13 & $10(77 \%)$ \\
Chest wall/neuromuscular & 5 & $4(80 \%)$ \\
CPO & 3 & $2(67 \%)$ \\
Obesity/hypoventilation and CPO & 2 & $1(50 \%)$ \\
Obesity/hypoventilation & 3 & 0 \\
Other & 2 & 0
\end{tabular}

COPD, chronic obstructive pulmonary disease; $\mathrm{CPO}$, cardiogenic pulmonary oedema. *Initial pH unavailable for two patients.

these patients are felt to be unsuitable for intubation/ventilation yet, interestingly, our results show that survival in this group does not differ significantly from that in patients presenting with an initial $\mathrm{pH}>7.26$. These results support earlier evidence that NIV is much less successful in patients who have alternative underlying diagnoses (eg, pneumonia). We conclude that the patient's underlying diagnosis may be more important than their admission $\mathrm{pH}$ in predicting success with NIV and survival to hospital discharge.

\section{P11 PATIENT SATISFACTION WITH AN ACUTE NON-INVASIVE VENTILATION (NIV) SERVICE}

K Walsh, AP Nataraja, MDL Morgan. Department of Respiratory Medicine, Glenfield Hospital, Leicester, UK

\section{doi:10.1136/thx.2009.127126k}

Background: The use of non-invasive ventilation (NIV) in addition to standard medical therapy is recommended in selected patients in acute type 2 respiratory failure. Patients should give consent where possible because lack of understanding and misperception of the treatment is an important factor in treatment failure. In order to gain a greater understanding about patient satisfaction, understanding of NIV and adherence to BTS recommendations on patient education, we surveyed previously treated patients in our tertiary referral unit.

Methods: A pre-piloted patient satisfaction survey was administered to patients who were identified from our acute respiratory failure database (table 1). Patients were contacted and surveyed by telephone in consultation with the local ethics committee.

Results: 204 patients receiving NIV between December 2007 and 2009 were identified from the database. Of these, 96 had died. Communication failure (language, deafness) prevented data collection from 13, and it was not possible to contact 46 (wrong or no number, no answer, nursing home residents). At total of 48/49 successfully contacted completed the survey (1 had no recollection of NIV treatment).

\section{Abstract P11 Table 1}

\begin{tabular}{lll}
\hline Patient satisfaction domains & Number & $\%$ \\
\hline Understood reason for NIV commencement & $29 / 48$ & 60 \\
Understood reason for discontinuation of NIV & $32 / 48$ & 67 \\
Understood what treatment involved & $24 / 48$ & 50 \\
Enough information available at time of NIV commencement & $37 / 48$ & 77 \\
Written information would be desirable before further & $27 / 48$ & 56 \\
treatment with NIV in the future & & \\
Not consented/no recollection of consent prior to treatment & $37 / 48$ & 77 \\
Satisfied with experience of treatment overall & $39 / 48$ & 81 \\
\hline
\end{tabular}

Conclusion: Patients were generally satisfied with the use of NIV as a treatment. They felt that they had received sufficient information regarding treatment initiation and discontinuation. Despite this, less than two-thirds understood why the treatment was started and only $50 \%$ understood what treatment would involve. Consent rates were low and no written information was offered. The following interventions may improve patient understanding and tolerance of NIV: trainee education on NIV and consent; patient information leaflet; and follow-up verbal confirmation of patient understanding It would be better to provide this patient information prior to need, perhaps during pulmonary rehabilitation.

\section{P12 RETROSPECTIVE EVALUATION OF HOME INITIATION FOR LONG- TERM NON-INVASIVE VENTILATORY SUPPORT}

JM Palmer, PD Hughes. Plymouth Hospitals NHS Trust, Plymouth, UK

doi:10.1136/thx.2009.127126|

Introduction: There are few data comparing hospital to domiciliary initiation of long-term non-invasive ventilatory (NIV) support. We have offered home initiation whenever feasible for some time and have compared our outcomes for these two groups.

Methods: Data were obtained by retrospective case note review and re-analysis of a patient satisfaction survey undertaken in July 2008. Results: 132 patients were set up over 5 years, $52 \%(n=69)$ at home and the rest in hospital. No adverse incidents were reported with home initiation. Mean length of stay for inpatient set-up was 6.7 days (range 1-30). Up to 450 bed-days were therefore saved as a result of domiciliary initiation. Overall concordance rate was $84.1 \%$ $(\mathrm{n}=111)$ with little difference in dropout rate between home and hospital initiation $(17.3 \%(n=12)$ vs $14.2 \%(n=9))$, similar to existing data for the outpatient department (82\% concordance) ${ }^{1}$ and hospital initiation (75\% and $97 \%$ concordance). ${ }^{23}$ Patients with chronic obstructive pulmonary disease were least likely to tolerate domiciliary initiation. While in hospital, those with motor neurone disease (MND) failed most frequently. Interestingly, patients with MND had excellent domiciliary success with only $8 \%(n=2)$ being non-concordant. Non-concordance does not appear to be strongly linked to support at home. Of those who did not tolerate NIV, 75\% $(n=9)$ of domiciliary set-ups and $78 \%(n=7)$ of inpatient set-ups lived with a partner or parent. Of those who lived alone, 78\% $(n=14)$ tolerated NIV. Inpatient set-up slightly favoured concordance in those who lived alone ( $81 \%$ vs $71 \%$ ). All patients initiated in $2007-8$ agreed or strongly agreed that they felt well supported by the service. Those being set up at home showed a greater tendency to strongly agree rather than just agree ( $75 \%$ vs $67 \%$ ).

Conclusion: Establishing NIV at home can be appropriate even for those with marked ventilatory failure. It does not affect concordance and supports patients while having the added advantage of decreasing bed usage and costs.

1. Chatwin, 2008.

2. Criner, 1999.

3. Simonds, 1996.

Abstract P12 Table 1 Mean (range) physiological data of patients commencing long-term non-invasive ventilatory support

\begin{tabular}{lcc}
\hline & Domiciliary initiation & Inpatient initiation \\
\hline $\mathrm{pH}$ & $7.38(7.29-7.44)$ & $7.38(7.22-7.48)$ \\
$\mathrm{PaCO}_{2}(\mathrm{kPa})$ & $7.25(4.94-10.3)$ & $8.61(4.80-14.8)$ \\
$\mathrm{PaO}_{2}(\mathrm{kPa})$ & $9.21(3.1-12.8)$ & $8.55(4.42-15.2)$ \\
$\mathrm{HCO}_{3}{ }^{-}(\mathrm{mmol} /)$ & $30.66(22.4-40.3)$ & $34.37(21.0-54.8)$ \\
$\mathrm{VC}(\mathrm{ml})$ & $1571(500-3230)$ & $1219(300-3930)$
\end{tabular}

$\mathrm{HCO}_{3}{ }^{-}$, bicarbonate; $\mathrm{PaCO}_{2}, \mathrm{PaO}_{2}$, arterial carbon dioxide and oxygen tensions; $\mathrm{VC}$, vital capacity. 